\title{
CONTROLE QUÍMICO DE DIGITARIA INSULARIS COM HERBICIDA COMBINADO A DIFERENTES ADJUVANTES
}

\author{
Gustavo Dias Menegasso ${ }^{1}$ \\ Edneia Santos de Oliveira Lourenço ${ }^{2}$ \\ Luis Augusto Schweig ${ }^{3}$
}

\begin{abstract}
Resumo: $O$ controle de plantas daninhas é uma prática importante para a obtenção de altos rendimentos em culturas agrícolas. O período de entressafra é favorável para a multiplicação de espécies de Digitaria insularis, que é considerada uma planta de difícil controle, onde se verifica a seleção e a resistência dessas plantas nas lavouras. Existe um número limitado de princípios ativos registrados no Brasil para o controle de Digitaria insularis. Clethodim é um herbicida sistêmico com alta eficiência, que pode ser utilizado no manejo de D. insularis. Para aumentar a eficiência deste herbicida são utilizados adjuvantes específicos que atuam de diversas formas na calda de pulverização. Dessa forma, o presente estudo teve como objetivo avaliar o controle químico da espécie D. insularis com o herbicida clethodim combinado à diferentes adjuvantes. O experimento foi conduzido em condições de campo, no município de São Miguel do Iguaçu-PR em 2018. O delineamento experimental utilizado foi o de blocos casualizados (DBC), em esquema fatorial composto por 6 doses do adjuvante Li700 ${ }^{\circledR}$ e 2 doses do adjuvante Lanzar ${ }^{\circledR}$, misturados a dose de $0,45 \mathrm{~L} \mathrm{ha}^{-1}$ do herbicida clethodim. As avaliações de eficácia agronômica foram efetuadas aos 7, 14 e 21 dias após a aplicação (DAA), empregando-se a escala percentual de controle. Os dados obtidos foram submetidos à ANOVA adotando o software Assistat ${ }^{\circledR}$ sendo as médias comparadas pelo teste de Tukey a $5 \%$ de significância e também por análise de regressão linear. Os resultados mostraram que o clethodim teve eficiência de controle classificada como boa (de 80 a 89\% de controle), patamar obtido com a combinação da maior concentração do adjuvante Li700 ${ }^{\circledR}$ enquanto o adjuvante Lanzar ${ }^{\circledR}$ não diferiu quanto à concentração na calda de pulverização. É alternativa viável a adição de Li $700^{\circledR}$ combinado ao adjuvante Lanzar $^{\circledR}$ para o controle pós emergente de Digitaria insularis, Contudo cabe salientar que o controle dessa espécie daninha deve ser realizado com adoção de medidas integradas e buscando minimizar o uso desenfreado dos herbicidas inibidores de ACCase no controle de Digitaria insularis, para garantir melhor eficiência e diminuir processos de resistência, de modo que que esta planta não continue a desenvolver-se, provocando danos às culturas de interesse agrícola.
\end{abstract}

Palavras-chave: resistência, clethodim, aderência foliar.

\section{CHEMICAL CONTROL OF DIGITARIA INSULARIS WITH HERBICIDE COMBINED WITH DIFFERENT ADJUVANTS}

\begin{abstract}
Weed control is an important practice for obtaining high yields in agricultural crops. The off-season is favorable for the multiplication of Digitaris insularis, Which is considered a very difficult control plant, where the selection and resistance of these plants in the crops is verified. There are a limited number of active principles registered in Brazil for the control of Digitaria insularis. Clethodim is a systemic herbicide with high efficiency, that can be used in the management of D. insularis. To enhance the efficiency of this herbicide specific adjuvants are used which act in various ways on the spray syrup. Thus, the present study aimed to evaluate the chemical control of D. insularis species with the herbicide clethodim combined with different adjuvants. The experiment was conducted in field conditions, in the municipality of São Miguel do Iguaçu-PR in 2018. The experimental design was a randomized complete block $(D B C)$, in a factorial scheme composed of 6 doses of the adjuvant Li700 ${ }^{\circledR}$ and 2 doses of the adjuvant Lanzar ${ }^{\circledR}$, mixed at a dose of 0.45 L ha-1 of the herbicide clethodim. The evaluations of agronomic effectiveness were performed at 7, 14 and 21 days after application (AAD), using the percentage control scale. The data were submitted to ANOVA using the Assistat ${ }^{\circledR}$ software, and the means were compared by the Tukey test at 5\% of significance and also by linear regression analysis. The results showed that the clethodim had control efficiency classified as good (from 80 to $89 \%$ of control), obtained with the combination of the highest Li700 ${ }^{\circledR}$ adjuvant concentration. In contrast, the adjuvant Lanzar ${ }^{\circledR}$ did not differ with respect to the concentration in
\end{abstract}

\footnotetext{
${ }^{1}$ Engenheiro Agrônomo, formado pelo Centro Universitário Dinâmica das Cataratas - UDC, campus Foz do Iguaçu - PR. Gustavomenegasso@hotmail.com

${ }^{2}$ Química formada pela Faculdade de Filosofia Ciências e Letras de Arapongas (1985). Doutora em Agronomia, área de concentração produção Vegetal, UNIOESTE (2014). Professora adjunta I do Centro Universitário Dinâmica das Cataratas - UDC. Tem experiência na área de Química, com ênfase em Química Orgânica, Analítica, Análise Ambiental atuando nos seguintes temas: Recursos Hídricos e Meio Ambiente, Remediação de Solo e Água por meio dos processos oxidativos avançados e-mails: Edneiasol@yahoo.com.br

${ }^{3}$ Engenheiro Agrônomo, formado pelo Centro Universitário Dinâmica das Cataratas - UDC, campus Foz do Iguaçu - PR. e-mail: Luyz_augusto@hotmail.com
} 
the spray mixture A viable alternative is the addition of combined Li700® to the Borde ${ }^{\circledR}$ adjuvant for the postemergence control of Digitaria insularis. However, it should be noted that the control of this weed species must be carried out with the adoption of integrated measures and to minimize the unrestrained use of the ACCase inhibitor herbicides in the control of Digitaria insularis, in order to guarantee better efficiency and reduce resistance processes, so that this plant does not continue to develop, causing damage to crops of agricultural interest.

Key-words: resistance, clethodin, foliar adherence

\section{Introdução}

A habilidade das plantas daninhas de sobreviverem em diversos ambientes é atribuída a sua agressividade competitiva, grande produção de sementes, facilidade de dispersão e longevidade. Essas plantas destacam-se entre as cultivadas devido à capacidade de aproveitamento de nutrientes do solo, sendo capazes de acumulá-los em quantidades maiores em seus tecidos (SILVA; SILVA, 2009).

Dentre as espécies a que tem sido mais preocupante na última década destaca-se o capimamargoso (Digitaria insularis) que é uma planta perene com capacidade de emergir e se desenvolver praticamente o ano inteiro em diferentes condições climáticas e em variados tipos de solo (LORENZI, 2008).

Uma forma de evitar perdas provocadas pelo capim-amargoso é realizar a dessecação, uma técnica de controle químico feita antes da semeadura da cultura comercial. A dessecação pode ser realizada com a utilização de produtos agroquímicos específicos para esta espécie gramínea (OLIVEIRA JÚNIOR; CONSTANTIN; INQUE, 2011).

A partir do desenvolvimento da soja transgênica com resistência ao herbicida gliphosate, o capim-amargoso se tornou de difícil controle com o uso do ingrediente ativo glifosato, base desta tecnologia transgênica (MONQUERO, 2014). Muitas vezes por um manejo inadequado e constante, cada vez mais se verifica a seleção e a resistência dessas plantas nas lavouras (OLIVEIRA et al., 2011).

Em estudo conduzido por Meschede et al. (2014) verificaram que o controle de capimamargoso na cultura da soja adotando herbicidas pós-emergentes alternativos ao glifosato, obtiveram controle superior ao glifosato. Dentre estes herbicidas estão o clethodim, o fluazifoppbuthyl, fenoxaprop-p-ethyl, o tepraloxydim, o paraquat, o haloxyfop-methyl e o imazapyr, cujas doses recomendadas conseguiram eliminar plantas já perenizadas. Alguns estudos demostraram a eficácia de herbicidas inibidores de ACCase na eliminação de capim-amargoso em fase inicial de crescimento (CORREIA; DURIGAN, 2009; MELO et al., 2012; BARROSO et al., 2014) e outros estudos verificaram o controle em plantas já em fase avançada de florescimento (PARREIRA et al., 2010; GEMELLI et al., 2012).

Este grupo de herbicidas possui ação em pós-emergência das plantas daninhas e apresenta características importantes, tais como ação seletiva a dicotiledôneas, como é o caso da soja, e alta 
eficácia no controle de gramíneas. Com isso, houve ampliação do uso dos herbicidas inibidores da ACCase, o que pode ter colaborado para a constatação do primeiro caso de resistência de $D$. insularis a herbicidas com esse mecanismo de ação no Brasil (HEAP, 2017).

A aplicação de clethodim para o controle de capim-amargoso indica que deve ser adicionado óleo adjuvante à calda de pulverização, visando melhorar a eficiência do ingrediente ativo sobre a espécie daninha. No entanto, existem muitos produtos que podem ser usados para promover maior cobertura das folhas e para aumentar a absorção dos herbicidas. Esses produtos são chamados de adjuvantes e são basicamente divididos em dois grupos: os modificadores das propriedades de superfície dos líquidos (surfactantes, espalhantes, umectante, detergentes, dispersantes e aderentes) e os aditivos (óleo mineral ou vegetal, sulfato de amônio e ureia) (VARGAS; ROMAN, 2006).

Diante do relato, este estudo objetivou avaliar o controle químico da espécie Digitaria insularis com o herbicida clethodim combinado à diferentes doses de adjuvantes.

\section{Material e Métodos}

O experimento foi conduzido em condições de campo, no município de São Miguel do Iguaçu (Figura 1), que é situado na Mesorregião Oeste do Estado do Paraná. Essa região pertence ao Terceiro Planalto Paranaense e faz fronteira com a Argentina e o Paraguai (IPARDES, 2003).

A área está situada numa altitude de 271 metros acima do nível do mar, na localização geográfica cujas coordenadas são Latitude $25^{\circ} 20^{\prime} 53$ "S e Longitude 54²14'16"O. O solo é classificado como Latossolo Vermelho Eutroférrico (Lve), de acordo com o Sistema Brasileiro de Classificação de Solos - SiBCS (EMBRAPA, 2013). A escolha da área foi em função da presença de plantas de capim-amargoso em estágio inicial de desenvolvimento, que infestavam totalmente a área.

O delineamento experimental utilizado foi o de blocos casualizados (DBC), em esquema fatorial composto por seis doses do adjuvante $\operatorname{Li} 700^{\circledR}$ e duas doses do adjuvante Lanzar ${ }^{\circledR}$, misturados a dose de $0,45 \mathrm{~L} \mathrm{ha}^{-1}$ do herbicida clethodim. No total, o experimento foi composto por 12 tratamentos com 4 repetições (Tabela 1). 
Tabela 1. Descrição dos tratamentos avaliados no experimento

\section{Tratamentos}

\begin{tabular}{|c|l|}
\hline T1 & 0,25\% Adjuvante Lanzar \\
T2 & 0,25\% Adjuvante Lanzar ${ }^{\circledR}+0,2 \%$ Adjuvante Li700 \\
T3 & 0,25\% Adjuvante Lanzar ${ }^{\circledR}+0,4 \%$ Adjuvante Li700 \\
T4 & 0,25\% Adjuvante Lanzar ${ }^{\circledR}+0,6 \%$ Adjuvante Li700 \\
T5 & 0,25\% Adjuvante Lanzar ${ }^{\circledR}+0,8 \%$ Adjuvante Li700 \\
T6 & 0,25\% Adjuvante Lanzar ${ }^{\circledR}+1,0 \%$ Adjuvante Li700 \\
T7 & 0,5\% Adjuvante Lanzar ${ }^{\circledR}$ \\
T8 & 0,5\% Adjuvante Lanzar ${ }^{\circledR}+0,2 \%$ Adjuvante Li700 \\
T9 & 0,5\% Adjuvante Lanzar ${ }^{\circledR}+0,4 \%$ Adjuvante Li700 \\
T10 & 0,5\% Adjuvante Lanzar ${ }^{\circledR}+0,6 \%$ Adjuvante Li700 \\
T11 & 0,5\% Adjuvante Lanzar ${ }^{\circledR}+0,8 \%$ Adjuvante Li700 \\
T12 & 0,5\% Adjuvante Lanzar ${ }^{\circledR}+1,0 \%$ Adjuvante Li700 \\
\hline
\end{tabular}

${ }^{1}$ Porcentagem em relação ao volume da calda (v.v.). ${ }^{2}$ Testemunha.

As parcelas foram constituídas por uma área de 3,0 m de largura e 4,0 metros de comprimento, totalizando uma área de $12,0 \mathrm{~m}^{2}$ por parcela e $576,0 \mathrm{~m}^{2}$ de área total destinada as 48 parcelas (Figura 1). Foram empregadas estacas para a demarcação de cada parcela.

Figura 1. Croqui de disposição

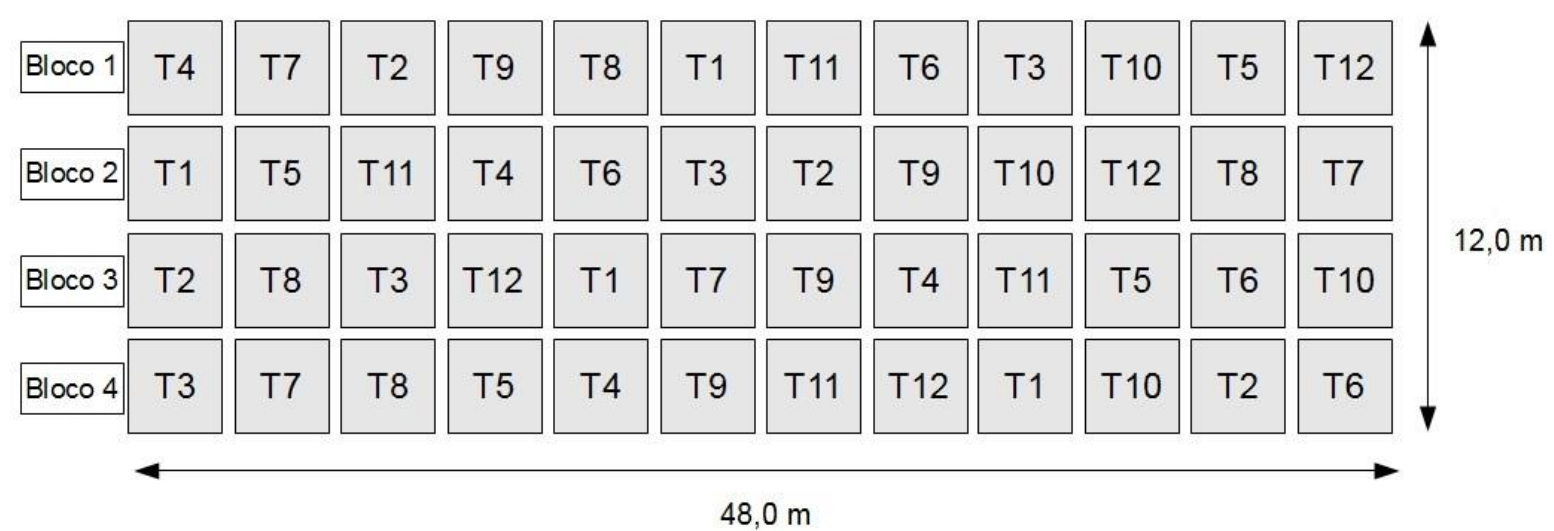

As parcelas foram constituídas por uma área de 3,0 m de largura e 4,0 metros de comprimento, totalizando uma área de $12,0 \mathrm{~m}^{2}$ por parcela e $576,0 \mathrm{~m}^{2}$ de área total destinada as 48 parcelas. Foram empregadas estacas para a demarcação de cada parcela. 
As aplicações dos tratamentos foram realizadas utilizando pulverizador costal de capacidade $5 \mathrm{~L}$, com pontas AXI 11002, distanciadas a 0,5 m entre si, mantido à pressão de trabalho de $3,0 \mathrm{kgf} \mathrm{cm}^{-2}$, que resultou em consumo de calda equivalente a $150 \mathrm{~L} \mathrm{ha}^{-1}$.

A aplicação foi realizada no momento que as condições climáticas estavam favoráveis, ou seja, com uma umidade relativa média de $65 \%$ e temperatura média de $28^{\circ} \mathrm{C}$. Na área a última precipitação ocorreu em 6 dias anterior à aplicação.

A avaliação de eficácia agronômica foram efetuadas aos 7, 14 e 21 dias após a aplicação (DAA), utilizando a escala percentual (Tabela 2), em que $0 \%$ representa ausência de controle, ou seja, quando o tratamento não apresenta nenhum resultado e $100 \%$ controle excelente, quando é feito o controle total do capim amargoso, levando em consideração quantidade de plantas por parcela (SBCPD, 1995).

Tabela 2. Descrição dos valores conceituais aplicado para avaliações visuais de controle aplicados na escala da Sociedade Brasileira da Ciência das Plantas Daninhas

\begin{tabular}{cc}
\hline Conceitos $(\boldsymbol{\%})$ & Descrição conceitual \\
\hline 90 a 100 & Controle excelente \\
80 a 89 & Controle bom \\
60 a 79 & Controle moderado \\
até 59 & Controle deficiente ou inexpressivo \\
0 & Ausência de controle \\
\hline
\end{tabular}

Fonte: SBCPD, 1995

As concentrações do adjuvante Li700 foram testadas por análise de regressão, em função de serem doses crescentes e quantitativas. As concentrações do adjuvante Lanzar foram testadas por teste de Tukey a 1 e $5 \%$ de probabilidade de erro. O software utilizado para análise foi o Assistat 7.7 beta.

\section{Resultados e Discussão}

A Tabela 3 mostra os resultados obtidos a partir da análise visual de variância, de controle de capim-amargoso nas avaliações feitas aos 7, 14 e 21 DAA. 
Tabela 3. Médias de controle de capim-amargoso (Digitaria insularis) aos 7, 14 e 21 DAA, submetido ao manejo químico com clethodim, combinado à diferentes concentrações dos adjuvantes Lanzar $^{\circledR}$ e $\operatorname{Li}_{700}{ }^{\circledR}$

\begin{tabular}{|c|c|c|c|c|}
\hline \multicolumn{2}{|c|}{ Tratamentos } & \multirow{2}{*}{$\begin{array}{c}7 \text { DAA } \\
20,0 \mathrm{aD}\end{array}$} & \multirow{2}{*}{$\frac{14 \text { DAA }}{40,0 \mathrm{aD}}$} & \multirow{2}{*}{$\frac{21 \text { DAA }}{70,0 \mathrm{aC}}$} \\
\hline $\mathrm{T} 1$ & $0,25 \%$ Lanzar $^{\circledR}$ & & & \\
\hline $\mathrm{T} 2$ & $0,25 \%$ Lanzar $^{\circledR}+0,2 \%$ Li700 $^{\circledR}$ & $22,5 \mathrm{aCD}$ & $40,0 \mathrm{aD}$ & $72,5 \mathrm{aB}$ \\
\hline $\mathrm{T} 3$ & $0,25 \%$ Lanzar $^{\circledR}+0,4 \%$ Li700 $^{\circledR}$ & $25,0 \mathrm{aC}$ & $45,0 \mathrm{aC}$ & $72,5 \mathrm{aB}$ \\
\hline $\mathrm{T} 4$ & $0,25 \%$ Lanzar $^{\circledR}+0,6 \%$ Li700 $^{\circledR}$ & $32,5 \mathrm{aB}$ & $46,3 \mathrm{bC}$ & $75,0 \mathrm{aB}$ \\
\hline T5 & $0,25 \%$ Lanzar $^{\circledR}+0,8 \%$ Li700 $^{\circledR}$ & $32,5 \mathrm{aB}$ & $52,5 \mathrm{~dB}$ & $80,0 \mathrm{aAB}$ \\
\hline T6 & $0,25 \%$ Lanzar $^{\circledR}+1,0 \%$ Li700 $^{\circledR}$ & $40,0 \mathrm{aA}$ & $65,0 \mathrm{aA}$ & $85,0 \mathrm{aA}$ \\
\hline $\mathrm{T} 7$ & $0,5 \%$ Lanzar $^{\circledR}$ & $20,0 \mathrm{aD}$ & $40,0 \mathrm{aE}$ & $72,5 \mathrm{aC}$ \\
\hline $\mathrm{T} 8$ & $0,5 \%$ Lanzar $^{\circledR}+0,2 \%$ Li7 $700^{\circledR}$ & $20,0 \mathrm{aD}$ & $42,5 \mathrm{aDE}$ & $70,0 \mathrm{aC}$ \\
\hline T9 & $0,5 \%$ Lanzar $^{\circledR}+0,4 \%$ Li700 $^{\circledR}$ & $22,5 \mathrm{aD}$ & $45,0 \mathrm{aD}$ & $72,5 \mathrm{aB}$ \\
\hline $\mathrm{T} 10$ & $0,5 \%$ Lanzar $^{\circledR}+0,6 \%{\text { Li } 700^{\circledR}}^{\circledR}$ & $27,5 \mathrm{aC}$ & $52,5 \mathrm{aC}$ & $77,5 \mathrm{aAB}$ \\
\hline $\mathrm{T} 11$ & $0,5 \%$ Lanzar $^{\circledR}+0,8 \%{\text { Li } 700^{\circledR}}^{\circledR}$ & $35,0 \mathrm{aB}$ & $57,5 \mathrm{aB}$ & $80,0 \mathrm{aAB}$ \\
\hline $\mathrm{T} 12$ & $0,5 \%$ Lanzar $^{\circledR}+1,0 \%$ Li700 $^{\circledR}$ & $40,0 \mathrm{aA}$ & $65,0 \mathrm{aA}$ & $85,0 \mathrm{aA}$ \\
\hline DMS & Fator $1\left(\right.$ Lanzar $\left.^{\circledR}\right)$ & 4,12 & 4,44 & 3,60 \\
\hline DMS & Fator $2\left(\operatorname{Li700}^{\circledR}\right)$ & 2,78 & 2,99 & 3,27 \\
\hline C.V. & & 13,4 & 11,7 & 10,2 \\
\hline Fcalc & Fator $1\left(\right.$ Lanzar $\left.^{\circledR}\right)$ & $0,1572^{\mathrm{ns}}$ & $0,0248 *$ & $0,1195^{\mathrm{ns}}$ \\
\hline Fcalc & Fator $2\left(\operatorname{Li700}^{\circledR}\right)$ & $0,0179^{*}$ & $0,0133 *$ & $0,0106^{*}$ \\
\hline
\end{tabular}

Médias seguidas pela mesma letra, na coluna, não diferem estatisticamente entre si pelo teste de Tukey a 5\% de probabilidade. Letras minúsculas referem-se às concentrações de Lanzar $^{\circledR}$ e letras maiúsculas referem-se às concentrações de $\mathrm{Li}_{700}{ }^{\circledR}$. C.V. = Coeficiente de variação. ns = não significativo.

Os resultados obtidos (Tabela 3) aos 7 DAA as concentrações do adjuvante Lanzar ${ }^{\circledR}$ na calda de pulverização não diferiram entre si, e somente na segunda avaliação, aos 14 DAA é que a concentração de $0,5 \%$ v.v. se sobressaiu em relação a concentração de $0,25 \%$ v.v., mas apenas quando utilizou-se das concentrações de $0,6 \%$ e $0,8 \%$ v.v. do adjuvante Li700 ${ }^{\circledR}$. Aos 21 DAA não se verificaram diferenças para as concentrações de Lanzar $^{\circledR}$.

Em estudo de Cavalieri (2017), em que se buscou determinar o efeito das formulações de glyphosate e adjuvantes na absorção do herbicida e controle de D. insularis resistente e 
suscetível, o adjuvante Lanzar $^{\circledR}$ foi um dos adjuvantes que apresentaram os menores valores para a tensão superficial (TS). Os valores menores de tensão superficial podem estar associados à maior eficácia de controle proporcionada por herbicidas (NAIRN; FORSTER; VAN LEEUWEN, 2015).

Maciel, Moraes e Balan (2011), ao avaliar os adjuvantes Triunfo 515®, Grap Super Gun ${ }^{\circledR}$ e Assist ${ }^{\circledR}(70 \mathrm{~mL}$ ha-1 $)$ promoveram incremento no controle de capim-amargoso (Digitaria insularis) para as formulações de glyphosate Polaris $®$ e Atanor $480 ®\left(2,25 \mathrm{~L} \mathrm{ha}^{-1}\right)$.

Quanto ao adjuvante $\mathrm{Li} 700^{\circledR}$, verificou-se que na concentração $1,0 \%$, o controle de capim-amargoso foi superior às demais concentrações testadas, sendo que o controle foi de $40 \%$ aos 7 DAA, 65\% aos 14 DAA e chegou a 85\% na última avaliação.

Em todos os períodos avaliados verificou-se que com o aumento na concentração do adjuvante $\mathrm{Li} 700^{\circledR}$, o controle de $D$. insularis também se mostrou mais acentuado, conforme ilustram as Figuras 4, 5 e 6. Em experimento conduzido por Correia e Resende (2016), apesar de não terem verificado diferenças em relação ao uso de diferentes adjuvantes na mistura com o herbicida clethodim, a ausência de adjuvantes à calda de aplicação não garantiu o controle da planta daninha, o que demostra a necessidade de se adicionar adjuvantes específicos na calda de aplicação em concentrações adequadas ou ainda, de forma combinada. Os resultados encontrados corroboram com Cunha (2003), que ressalta que a utilização de adjuvantes beneficia o desempenho da aplicação, favorecendo o espalhamento e molhamento foliar, além da redução da deriva.

Ryckaert (2007) relata ainda que a incorporação de adjuvantes à calda de pulverização favorece o resultado das aplicações, pois eles proporcionam uma maior a aderência e absorção do ingrediente ativo sobre a superfície.

Estes resultados discordam dos obtidos por Dan et al. (2010) por não terem constatado benefícios da adição dos adjuvantes multifuncionais à calda de aplicação de quatro formulações de glyphosate (1080 g i.a. ha ${ }^{-1}$ ), para o controle de capim amargoso (D. insularis) em estágio de desenvolvimento vegetativo.

Na Figura 2 é possível observar os resultados obtidos a partir da análise de regressão, de controle de capim-amargoso na avaliação feita aos 7 DAA. 
Figura 2. Controle de capim-amargoso aos 7 DAA em função de diferentes concentrações de adjuvante Lanzar $^{\circledR}$ e Li700 ${ }^{\circledR}$ misturados ao herbicida

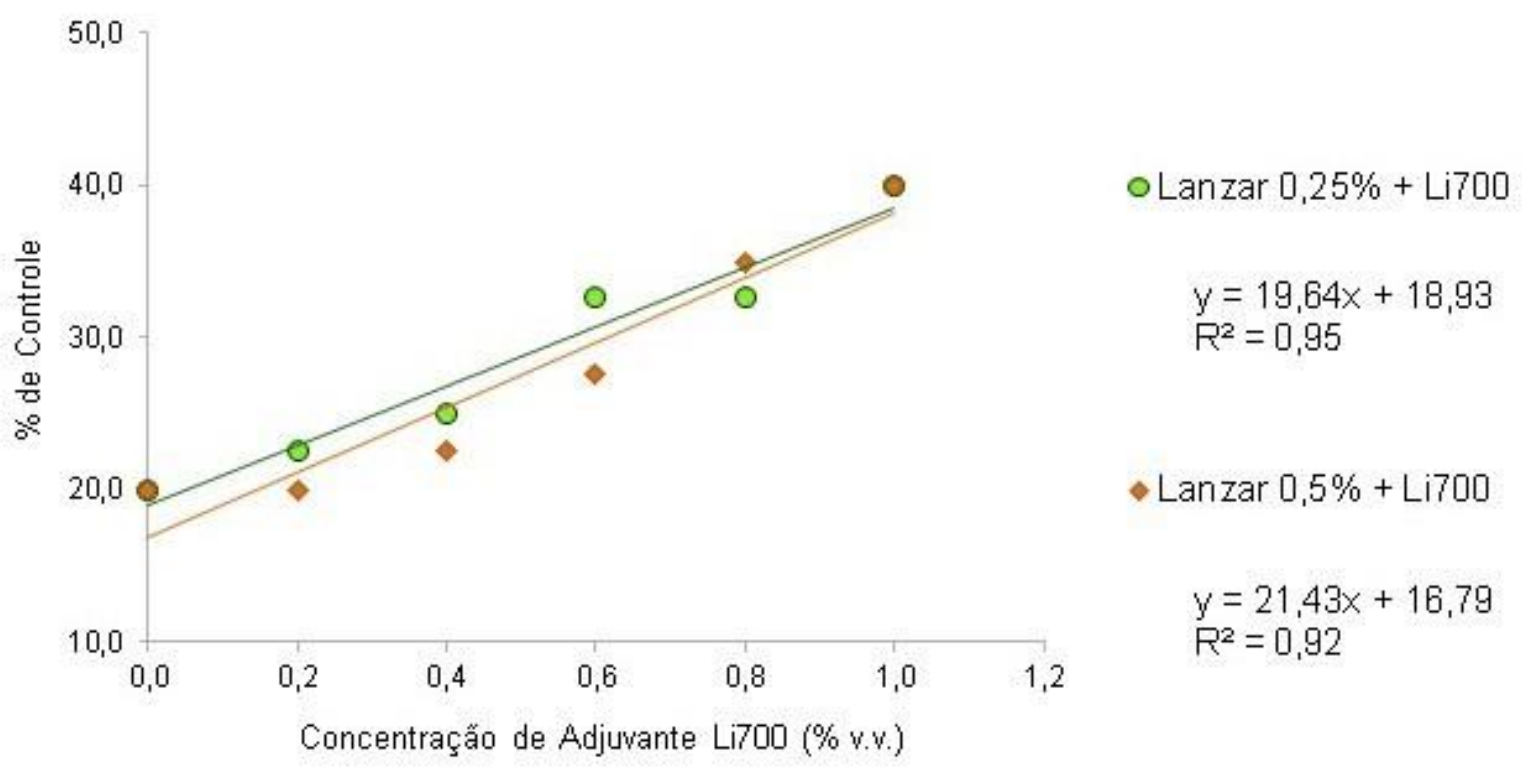

Conforme a avaliação feita aos 7 DAA demonstra, o melhor tratamento chegou a $40 \%$ de controle, com as doses 0,25\% de Lanzar e 1,0\% de li700 juntamente com 0,5\% de Lanzar e 1,0\% de $\operatorname{Li} 7000$.

Observou-se na primeira análise da avaliação que os resultados só se alteravam conforme o aumento da dose de Li700, independente da concentração de Lanzar, o que pode ser explicado pelas características do produto de maior molhamento, facilitando a absorção do Herbicida.

Em estudo conduzido por Cunha et al. (2017), em que avaliaram o comportamento da associação entre 15 produtos fitossanitários e quatro adjuvantes de uso agrícola na tensão superficial, $\mathrm{pH}$ e condutividade elétrica da calda, o adjuvante Li700 mostrou ser o produto com maior capacidade de reduzir o potencial hidrogeniônico $(\mathrm{pH})$ da calda de aplicação. Segundo os autores, o pH da calda de pulverização influencia na absorção dos produtos dissolvidos na calda pelo tecido foliar, uma vez que o $\mathrm{pH}$ interfere na constante de dissociação de inúmeras moléculas.

Ronke Júunior (2017) destaca que a associação dos adjuvantes a calda objetiva que os adjuvantes ampliem a eficiência, porém, a interação adjuvante e agrotóxico é um procedimento complexo, que abrange muitos aspectos físicos, fisiológicos e químicos, podendo variar conforme cada circunstância testada.

A Figura 3 mostra os resultados obtidos a partir da análise de regressão de controle de capim-amargoso na avaliação feita aos 14 DAA em função de diferentes concentrações de 
adjuvante $\mathrm{L}$ Lanzar $^{\circledR}$ e Li700 $^{\circledR}$ misturados ao herbicida clethodim.

Figura 3. Controle de capim-amargoso aos 14 DAA em função de diferentes concentrações de adjuvante Lanzar $^{\circledR}$ e Li700 ${ }^{\circledR}$ misturados ao herbicida clethodim.

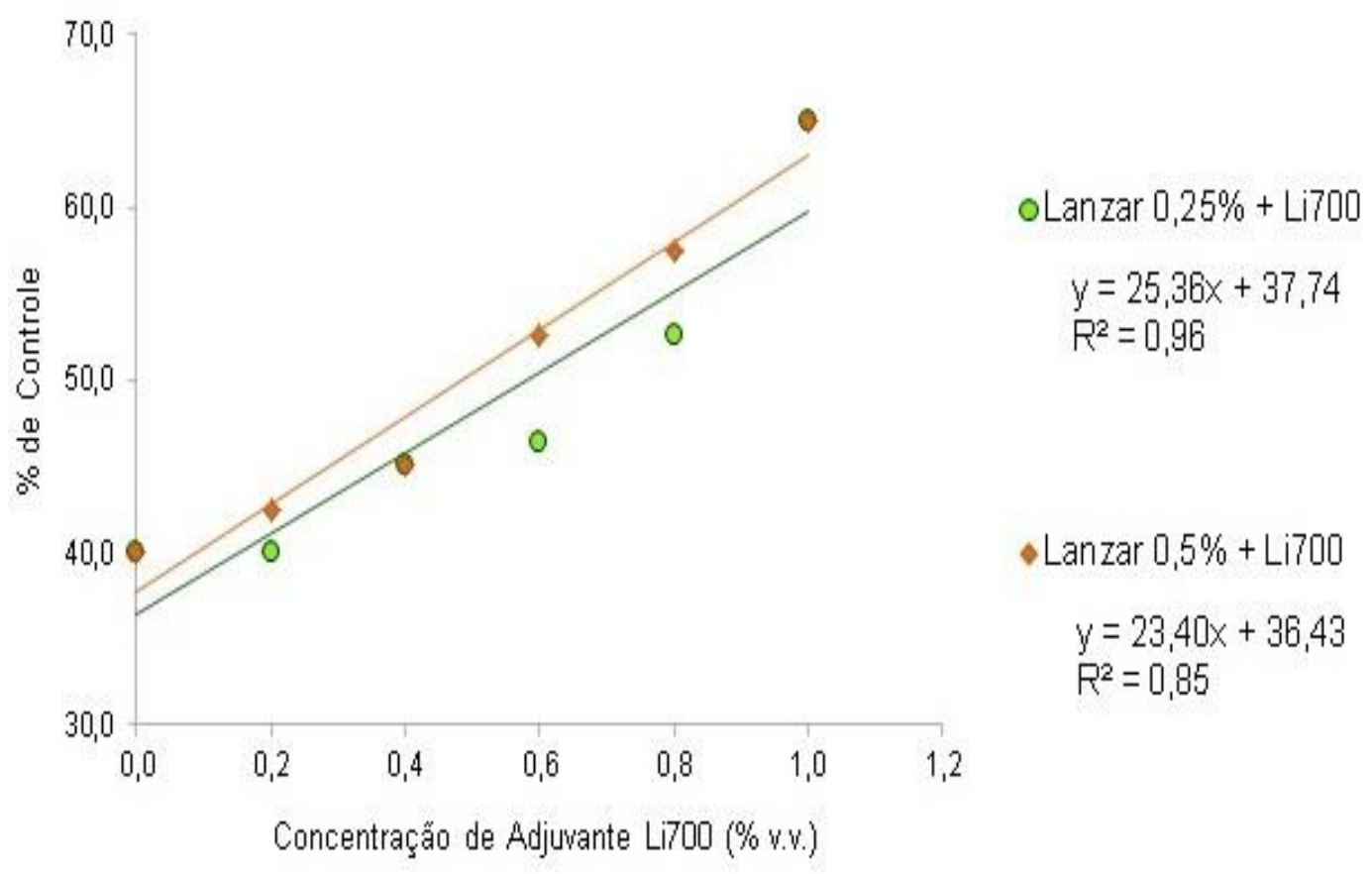

Pode-se analisar que aos 14 DAA os tratamentos com maior eficácia continuavam sendo os de 1,0\% de Li700m, controlando até $65 \%$ das plantas de capim amargoso. Nota-se também que os 14 DAA o Lanzar na concentração de $0,5 \%$ aponta um resultado melhor do que na concentração de $0,25 \%$.

Peressin, Vitória Júnior e Perecin (1997), avaliaram que o efeito dos adjuvantes no controle de $E$. indica, $D$. horizontalis, monocotiledôneas e $M$. verticillata foi significativo, indicando que sua utilização, de maneira geral, também favoreceu o controle. Em estudo realizado por Ferreira, Roman e Lemos (2010), o adjuvante Li 700 melhorou consideravelmente a distribuição do herbicida, favorecendo um controle mais satisfatório.

A Figura 4 mostra os resultados obtidos a partir da análise de regressão de controle de capim-amargoso na avaliação feita aos 21 DAA em função de diferentes concentrações de adjuvante $\mathrm{L} \mathrm{Lanzar}^{\circledR}$ e $\mathrm{Li} 700^{\circledR}$ misturados ao herbicida clethodim. 
Figura 4. Controle de capim-amargoso aos 21 DAA em função de diferentes concentrações de adjuvante Lanzar $^{\circledR}$ e $\operatorname{Li}_{700}{ }^{\circledR}$ misturados ao herbicida clethodim.

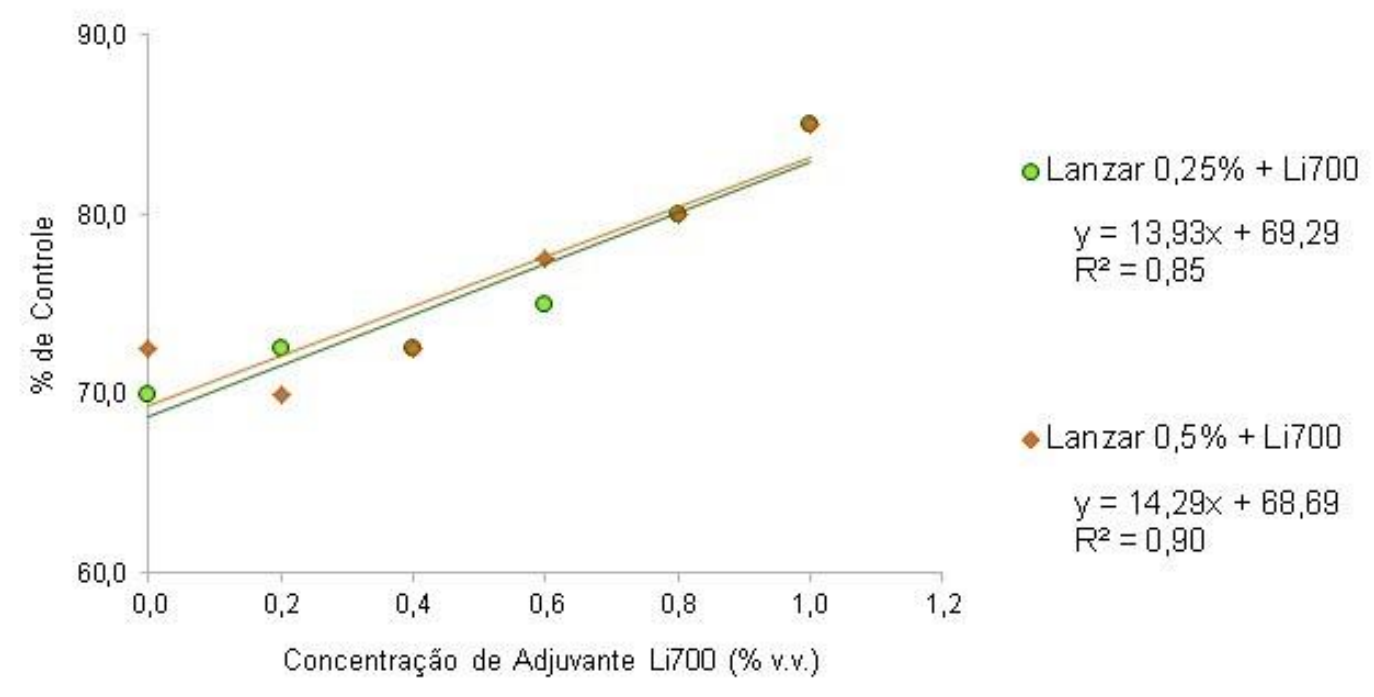

De forma geral, não se observou controle total da espécie $D$. insularis aos 21DAA, resultado este semelhante ao de Fornarolli (2011), que alcançaram controle inferior a $100 \%$ utilizando os mesmos métodos de controle de Digitaria insularis. Entretanto, resultados obtidos por Adegas (2010) são contraditórios aos do presente estudo já que estes autores obtiveram 95\% de controle aos 21 DAA utilizando o herbicida clethodim em plantas com $38 \mathrm{~cm}$ de altura.

Os resultados (Figura 4) mostram que em função do aumento na concentração do adjuvante Li700 ${ }^{\circledR}$, melhora-se o controle de $D$. insularis de forma linear, o que indica que em maiores concentrações deste adjuvante, independente da dose de Lanzar $^{\circledR}$ adicionada à calda, é provável que se obtenha controle total da planta daninha. Desta forma, são necessários estudos sobre a utilização de concentrações de adjuvantes que sejam superiores as recomendadas na bula do herbicida clethodim, visando satisfazer as necessidades da calda de aplicação para se obter controle eficiente sobre esta espécie.

Como as plantas de capim-amargoso apresentavam altura superior a $40 \mathrm{~cm}$ no momento da aplicação dos tratamentos, o controle químico atingiu o máximo de $85 \%$, o que demonstra as limitações do herbicida clethodim no controle de plantas em elevado grau de crescimento. Fato este também relatado por Gemelli et al. (2012) que destacou como ponto chave no incremento da ocorrência de $D$. insularis que, uma vez que a planta esteja estabelecida com o início da formação dos rizomas e posterior formação de grandes touceiras, ela se torna de difícil controle.

Segundo Zobiole (2016) nos estágios mais avançados de desenvolvimento do capimamargoso, com plantas que já se apresentam perenizadas, é necessário o uso de aplicações 
sequenciais de herbicidas para o controle desta espécie, além da associação entre herbicidas com mecanismos de ação distintos. Assim, o emprego de adjuvantes à calda de aplicação é prática indispensável para aumentar a eficiência do controle de D. insularis.

Procópio et al. (2006) ressaltaram-se que para o manejo dessa espécie de planta daninha em estágio de desenvolvimento avançado é importante obter eficiência máxima de controle na operação de dessecação de pré-semeadura, objetivando inviabilizar a ocorrência de rebrotes e/ou reinfestação da mesma após a semeadura da cultura de interesse.

\section{Considerações Finais}

No presente estudo, a combinação dos adjuvantes na calda de aplicação se mostrou eficiente para o controle em pós emergência de Digitaria insularis, atingindo-se um nível de controle classificado como bom (de 80 a $89 \%$ de controle).

O melhor patamar de controle foi obtido com o aumento na concentração do adjuvante Li700 $^{\circledR}$ a 1,0\% combinado ao adjuvantes Lanzar $^{\circledR}$ nas concentrações de 0,25 e 0,50\%, sendo portanto alternativa viável a adição de Li700 $^{\circledR}$ combinado ao adjuvante Lanzar ${ }^{\circledR}$ para o controle pós emergente de Digitaria insularis.

O manejo de controle de capim-amargoso, visando a sua eliminação em lavouras de interesse comercial deve ser realizado seguindo-se as recomendações técnicas já consolidadas na teoria e na prática, tal como a utilização do controle químico com herbicidas, como é caso do ingrediente ativo clethodim.

Contudo, cabe salientar que o controle dessa espécie daninha deve ser realizado com adoção de medidas integradas e buscando minimizar o uso desenfreado dos herbicidas inibidores de ACCase no controle de Digitaria insularis, atentando ao ponto de controle da espécie para garantir melhor eficiência e diminuir processos de resistência, de modo que que esta planta não continue a desenvolver-se, provocando danos às culturas de interesse agrícola.

\section{Referências}

ADEGAS, F.S. et al. Alternativas de controle químico de Digitaria insularis resistente ao herbicida glyphosate. In: CONGRESSO BRASILEIRO DA CIÊNCIA DAS PLANTAS DANINHAS, 27. Ribeirão Preto, SP. 2010. Resumos expandidos.... Ribeirão Preto: SBCPD, n.161, 2010b

BARROSO, A. A. M.; ALBRECHT, A. J. P.; REIS, F. C.; FILHO, R. V. Interação entre herbicidas inibidores de ACCase e diferentes formulações de glyphosate no controle de capimamargoso. Planta Daninha, v. 32, n. 3, p. 619-627, 2014.

CAVALIERI, J. D. Resistência de populações de Digitaria insularis (L). Fedde a herbicidas: frequência de ocorrência e interações entre formulações de glyphosate e adjuvantes. Botucatu, 2015. 101p. Tese (Doutorado em Agronomia)-Universidade Estadual Paulista, São Paulo, 2015. 
CORREIA, N. M.; DURIGAN, J. C. Manejo químico de plantas adultas de Digitaria insularis com glyphosate isolado e em mistura com chlorimuron-ethyl ou quizalofop-p-tefuril em área de plantio direto. Bragantia, v. 68, n. 3, p. 689-697, 2009.

CORREIA, N. M.; RESENDE, I. Associação de adjuvantes ao herbicida clethodim e o controle de Digitaria insularis. In: Congresso Brasileiro da Ciência das Plantas Daninhas, 30, Anais. Curitiba: SBCPD, 2016. p. 761.

CUNHA, J. P. A. R.; ALVES, G. S. MARQUES, R. S. Tensão superficial, potencial hidrogeniônico e condutividade elétrica de caldas de produtos fitossanitários e adjuvantes. Revista Ciência Agronômica, Fortaleza, v. 48, n. 2, p. 261-270, 2017.

CUNHA, J. P. A. R.; TEIXEIRA, M. M.; COURY, J. R.; FERREIRA, R. L. Avaliação de estatégias para redução da deriva de agrotóxicos em pulverizações hidráulicas. Planta Daninha, v. 21, n. 2, p. 325-332, ago. 2003

DAN, H. A.; BARROSO, A. L. L.; DAN, L. G. M.; FINOTTE, T. R.; FELDKIRCHER, C. A. Adjuvantes multifuncionais associados ao herbicida glyphosate no controle de Digitaria insularis. Global Science and Technology, v.3, n.2, p.30-38, 2010.

EMBRAPA. Empresa Brasileira de Pesquisa Agropecuária. Sistema brasileiro de classificação de solos. 3. ed. Brasília, 2013.

FERREIRA, M. C.; ROMAN, R. A. A.; LEMOS, R. E. Efeito de adjuvante na distribuição volumétrica de glifosate por pontas de pulverização de jato plano com indução de ar. In: XXVII Congresso Brasileiro da Ciência das Plantas Daninhas, 2010, Ribeirão Preto - SP. Anais do XXVII Congresso Brasileiro da Ciência das Plantas Daninhas, 2010.

FORNAROLLI, D. A. GAZZIERO, D. L. P.; BONOTTO, A. T.; SANTOS, B. C. dos; DEBASTIANI, R.; BANDEIRA, S. A. E. Manejo de biotípos de Digitaria insularis resistente ao herbicida glifosato. In: III SIMPÓSIO INTERNACIONAL SOBRE GLYPHOSATE, Botucatu, SP, 2011. Resumos... Botucatu:UNESP, 2011

MONQUERO, P. A. Manejo de plantas daninhas em culturas agrícolas. São Carlos: RiMa Editora, 2014.

HEAP, I. International Survey of Herbicide Resistant Weeds. 2014. Disponível em: <http://www.weedscience.org/In.asp>. Acesso em 02 jun. 2018.

GEMELLI, A.; OLIVEIRA JÚNIOR, S.; CONSTANTIN, J. C.; BRAZ, G. B. P.; JUMES, T. M. C.; OLIVEIRA NETO, A. M.; DAN, H. A.; BIFFE, D. F. Aspectos da biologia de Digitaria insularis resistente ao glyphosate e implicações para o seu controle. Revista Brasileira de Herbicidas, v. 11, n. 2, p. 231-240, 2012.

IPARDES. Instituto Paranaense de Desenvolvimento Econômico e Ssocial. Leituras regionais: mesorregião geográfica oeste paranaense. Curitiba: IPARDES: BRDE, 2003.

LORENZI, H. Plantas daninhas no Brasil: terrestres, aquáticas, parasitas e tóxicas. 4.ed. Nova Odessa: Plantarum, 2008.

MACIEL, C. D. G.; MORAES, D. W.; BALAN, M. G. Associação de adjuvantes com herbicidas na dessecação e no controle em pósemergência de plantas daninhas na cultura do trigo. Rev. Bras. Herb., v. 10, n. 3, p. 243-256, set./dez. 2011.

MELO, M. S. C.; ROSA, L. E.; BRUNHARO, C. A. C.; NICOLAI, M.; CHRISTOFFOLETTI, P. J. Alternativas de controle para capim-amargoso (Digitaria insularis) resistente ao glifosato. Anais. In: Congresso Brasileiro da Ciência das Plantas Daninhas, 27, 2010, Ribeirão Preto.

MELO, M. S. C.; ROSA, L. E.; BRUNHARO, C. A. C. G.; NICOLAI, M.; CHRISTOFFOLETI, P. J. Alternativas para o controle químico de capim-amargoso (Digitaria insularis) resistente ao 
glyphosate. Revista Brasileira de Herbicidas, v. 2, n. 11, p. 195-203, 2012.

MESCHEDE, D. K. A importância no controle do capim amargoso (D. insularis): "estudos de casos”. In: Reunião de Pesquisa de Soja, 34, 2014, Londrina. Palestra. Londrina: Embrapa Soja, 2014.

NAIRN, J. J.; FORSTER, W. A.; VAN LEEUWEN, R. M. Effect of solution and leaf surface polarity on droplet spread area and contact angle. Pest Management Science, v. 72, n. 3, p. 551$557,2015$.

OLIVEIRA JUNIOR., R. S.; CONSTANTIN, J.; INQUE, M. H. Biologia e manejo de plantas daninhas. Curitiba: Ed. Omnipax, 2011.

PARREIRA, M. C.; ESPANHOL, M.; DUARTE, D. J.; CORREIA, N. M. Manejo químico de Digitaria insularis em área de plantio direto. Revista Brasileira de Ciências Agrárias, v. 5, n. 1, p. 13- 17, 2010.

PERESSIN, V. A. VICTÓRIA FILHO, R.; PERECIN, D. Misturas de herbicidas: efeitos de adjuvantes no controle de plantas infestantes na cultura da soja. Bragantia, v. 56, n. 1, p. 103116, 1997.

PROCÓPIO, S. O.; PIRES, F. R.; MENEZES, C. C. E.; BARROSO, A. L. L.; MORAES, R. V.; SILVA, M. V. V.; QUEIROZ, R. G.; CARMO, M. L. Efeitos de dessecantes no controle de plantas daninhas na cultura da soja. Planta Daninha, v. 24, n. 1, p. 193-197, 2006.

RONKE JUNIOR, E. E. Interação de adjuvantes e herbicida inibidores de accase no controle de digitaria insularis. 2017. Monografia. (Graduação em Engenharia Agronômica)- Centro Universitário Dinâmica das Cataratas, Foz do Iguaçu, 2017.

RYCKAERT, B.; SPANOGHE, P.; HAESAERT, G.; HEREMANS, B.; ISEBAERT, S.; STEURBAUT, W. Quantitative determination of the influence of adjuvants on foliar fungicide residues. Crop Protec., v. 26, n. 10, p. 1589-1594, 2007.

SILVA, A. A.; SILVA, J. F. Tópicos em Manejo de Plantas Daninhas. 1. ed. Viçosa: UFV, 2009.

SBCPD. Sociedade Brasileira da Ciência das Plantas Daninhas. Procedimentos para instalação, avaliação e análise de experimentos com herbicidas. Londrina: SBCPD, 1995.

VARGAS, L.; ROMAN, E. S. Conceitos e aplicações dos adjuvantes. Passo Fundo: Embrapa Trigo, 2006.

ZOBIOLE, L. H. S.; KRENCHINSKI, F. H.; ALBRECHT, A. J. P.; PEREIRA, G.; LUCIO, F. R.; ROSSI, C.; RUBIN, R. S. Controle de capim-amargoso perenizado em pleno florescimento. Revista Brasileira de Herbicidas, v. 15, n. 2, p. 157-164, 2016. 